\title{
EXISTENCE OF SOLUTIONS OF STRONGLY NONLINEAR ELLIPTIC EQUATIONS IN $\mathbf{R}^{N}$
}

\author{
A. BENKIRANE and M. KBIRI ALAOUI
}

\begin{abstract}
The paper is dedicated to the existence of local solutions of strongly nonlinear equations in $\mathbb{R}^{N}$ and the Orlicz spaces framework is used.
\end{abstract}

\section{Introduction}

Let $A$ be the Leray-Lions operator given by $A(u)=-\operatorname{div} a(., u, \nabla u)$ where $p \in] 1, N], \frac{1}{p}+\frac{1}{p^{\prime}}=1$ and where $a: \mathbf{R}^{N} \times \mathbf{R} \times \mathbf{R}^{N} \rightarrow \mathbf{R}^{N}$ is a Carathéodory function satisfying for a.e. $x \in \mathbf{R}^{N}$, for all $s \in \mathbf{R}, \xi, \xi^{*} \in$ $\mathbf{R}^{N}$ with $\xi \neq \xi^{*}$ :

$$
\begin{gathered}
|a(x, s, \xi)| \leq \beta\left(c(x)+b(x)|s|^{p_{-1}}+d(x)|\xi|^{p_{-} 1}\right), \\
{\left[a(x, s, \xi) \_a\left(x, s, \xi^{*}\right)\right]\left[\xi_{-} \xi^{*}\right]>0,} \\
\nu|\xi|^{p} \leq a(x, s, \xi) \xi
\end{gathered}
$$

where $c(x) \in L_{l o c} p^{\prime}\left(\mathbf{R}^{N}\right), c \geq 0 ; b(x), d(x)$ are locally bounded functions, $\beta \in \mathbf{R}^{+}$and $\nu>0$. On the other hand, $g: \mathbf{R}^{N} \times \mathbf{R} \rightarrow \mathbf{R}$ is a Carathéodory function such that for a certain constant $\delta>0$ :

$$
g(x, s) \text { signs } \geq|s|^{\delta},
$$

holds for all $s \in \mathbf{R}$, a.e $x \in \mathbf{R}^{N}$. Moreover, for every $t>0$,

$$
\sup _{|s| \leq t}|g(., s)| \in L_{l o c}^{1}\left(\mathbf{R}^{N}\right)
$$

2000 Mathematics Subject Classification: 35J60.

Servicio de Publicaciones. Universidad Complutense. Madrid, 2001 
a.e. $x \in \mathbf{R}^{N}$.

We consider the following nonlinear equation

$$
A(u)+g(., u)=f \text { in } \mathbf{R}^{N}, f \in L_{l o c}^{1}\left(\mathbf{R}^{N}\right) .
$$

We say that $u$ is a weak solution of (1.6) if it satisfies:

$$
\left\{\begin{array}{l}
u \in W_{l o c}^{1,1}\left(\mathbf{R}^{N}\right), a(., u, \nabla u) \in L_{l o c}^{1}\left(\mathbf{R}^{N}\right), g(., u) \in L_{l o c}^{1}\left(\mathbf{R}^{N}\right) \\
A(u)+g(., u)=f \text { in } \mathcal{D}^{\prime}\left(\mathbf{R}^{N}\right)
\end{array}\right.
$$

By combining [10] and [11], we can deduce the existence of weak solutions $u$ for (1.6), with the following regularity:

$u \in W_{l o c}^{1, q}\left(\mathbf{R}^{N}\right)$ for every $q<\bar{q}=\frac{N\left(p \_1\right)}{N_{-} 1}$ if $\delta>p-1$ and $p_{0}=2-\frac{1}{N}<$ $p \leq N$.

$u \in W_{l o c}^{1, q}\left(\mathbf{R}^{N}\right)$ for every $q<q_{1}=\frac{p \delta}{\delta+1}$ if $\delta>\bar{q}^{*}$ and $p_{0}<p<N$ or $\delta(p-1)>1$ and $1<p \leq p_{0}$.

It is our purpose in this paper to prove the limiting regularity $W_{l o c}^{1, \bar{q}}\left(\mathbf{R}^{N}\right)$ and $W_{l o c}^{1, q_{1}}\left(\mathbf{R}^{N}\right)$ (which is not reached in general ) of weak solutions of (1.6), when we replace the conditions (1.1) and (1.3) by the following:

$$
\begin{gathered}
|a(x, s, \xi)| \leq \beta\left(c(x)+b(x)|s|^{p-1} \log ^{\alpha p}(e+|s|)\right. \\
\left.+d(x)|\xi|^{p \_1} \log ^{\alpha p}(e+|\xi|)\right) \\
\nu|\xi|^{p} \log ^{\alpha p}(e+|\xi|) \leq a(x, s, \xi) \xi
\end{gathered}
$$

a.e. $x \in \mathbf{R}^{N}, \forall s \in \mathbf{R}$ and $\xi, \xi^{*} \in \mathbf{R}^{N}, \xi \neq \xi^{*}$. With $c, b(x), d(x), \beta, \nu$ as above, $1<p \leq N$, and $\alpha>\frac{1}{p}$.

It is to be noticed that our study covers the regularity $W_{l o c}^{1, N}\left(\mathbf{R}^{N}\right)$.

We prove also that the condition $\alpha>1 / p$ is necessary to obtain the limiting regularity $W_{l o c}^{1, \bar{q}}\left(\mathbf{R}^{N}\right)$ for the solutions given by theorem 2.1.

The case $g(., s)$ signs $\geq 0$ was studied in [8] with second member measure and $\Omega$ bounded.

For other strongly nonlinear equations in Orlicz spaces see [7], [5], [6], [15], [16]

\section{Preliminaries}

We list some well known results about Orlicz and Orlicz-Sobolev spaces. 
2.1. Let $M: \mathbf{R}^{+} \rightarrow \mathbf{R}^{+}$be an N-function, i. e. $M$ is continuous, convex with $M(t)>0$ for $t>0, \frac{M(t)}{t} \rightarrow 0$ as $t \rightarrow 0$ and $\frac{M(t)}{t} \rightarrow \infty$ as $t \rightarrow \infty$.

Equivalently, $M$ admits the representation: $M(t)=\int_{0}^{t} a(s) d s$ where $a: \mathbf{R}^{+} \rightarrow \mathbf{R}^{+}$is nondecreasing, right continuous, with $a(0)=0, a(t)>0$ for $t>0$ and $a(t) \rightarrow \infty$ as $t \rightarrow \infty$.

The N-function $\bar{M}$ conjugate to $M$ is defined by $\bar{M}(t)=\int_{0}^{t} \bar{a}(s) d s, \bar{a}$ : $\mathbf{R}^{+} \rightarrow \mathbf{R}^{+}$is given by $\bar{a}(t)=\sup \{s: a(s) \leq t\}$ (see [1], [17]).

The N-function is said to satisfy the $\Delta_{2}$ condition if, for some $k>0$ :

$$
M(2 t) \leq k M(t) \quad \forall t \geq 0
$$

when holds only for $t \geq t_{0}>0$ then $M$ is said to satisfy the $\Delta_{2}$ condition near infinity .

We will extend these $\mathrm{N}$-functions into even functions on all $\mathbf{R}$.

2.2. Let $\Omega$ be an open subset of $\mathbf{R}^{N}$. The Orlicz class $K_{M}(\Omega)$ (resp.the Orlicz space $L_{M}(\Omega)$ )is defined as the set of (equivalences classes of)real valued measurable functions $u$ on $\Omega$ such that:

$\int_{\Omega} M(u(x)) d x<+\infty\left(\operatorname{resp} \cdot \int_{\Omega} M\left(\frac{u(x)}{\lambda}\right) d x<+\infty\right.$ for some $\left.\lambda>0\right)$.

$L_{M}(\Omega)$ is a Banach space under the norm:

$$
\|u\|_{M, \Omega}=\inf \left\{\lambda>0: \int_{\Omega} M\left(\frac{u(x)}{\lambda}\right) d x \leq 1\right\}
$$

and $K_{M}(\Omega)$ is a convex subset of $L_{M}(\Omega)$.

The closure in $L_{M}(\Omega)$ of the set of bounded measurable functions with compact support in $\bar{\Omega}$ is denoted by $E_{M}(\Omega)$.

The equality $E_{M}(\Omega)=L_{M}(\Omega)$ holds if and only if $M$ satisfies the $\Delta_{2}$ condition, for all $t$ or for $t$ large according to whether $\Omega$ has infinite measure or not .

The dual of $E_{M}(\Omega)$ can be identified with $L_{\bar{M}}(\Omega)$ by means of pairing $\int_{\Omega} u(x) v(x) d x$ and the dual norm on $L_{\bar{M}}(\Omega)$ is equivalently to $\|u\|_{\bar{M}}, \Omega$.

The space $L_{M}(\Omega)$ is reflexive if and only if $M$ and $\bar{M}$ satisfy the $\Delta_{2}$ condition, for all $t$ or for $t$ large according to whether $\Omega$ has infinite measure or not .

2.3. We now turn to the Orlicz-Sobolev space. $W^{1} L_{M}(\Omega)$ (resp. $\left.W^{1} E_{M}(\Omega)\right)$ is the space of all functions such that $u$ and its distributional derivatives up to order 1 lie in $L_{M}(\Omega)\left(\right.$ resp. $\left.E_{M}(\Omega)\right)$. 
It is a Banach space under the norm :

$$
\|u\|_{1, M, \Omega}=\sum_{|\alpha| \leq 1}\left\|D^{\alpha} u\right\|_{M, \Omega}
$$

Thus, $W^{1} L_{M}(\Omega)$ and $W^{1} E_{M}(\Omega)$ can be identified with subspaces of the product of

$\mathrm{N}+1$ copies of $L_{M}(\Omega)$. Denoting this product by $\prod L_{M}$, we will use the weak topologies $\sigma\left(\prod L_{M}, \prod E_{\bar{M}}\right)$ and $\sigma\left(\prod L_{M}, \prod L_{\bar{M}}\right)$.

The space $W_{0}^{1} E_{M}(\Omega)$ is defined as the (norm) closure of the Schwartz space $\mathcal{D}(\Omega)$ in $W^{1} E_{M}(\Omega)$ and the space $W_{0}^{1} L_{M}(\Omega)$ as the $\sigma\left(\prod L_{M}, \prod E_{\bar{M}}\right)$ closure of $\mathcal{D}(\Omega)$ in $W^{1} L_{M}(\Omega)$.

Let $W^{-1} L_{\bar{M}}(\Omega)\left(\right.$ resp. $W^{-1} E_{\bar{M}}(\Omega)$ )denote the space of distributions on $\Omega$ which can be written as sums of derivatives of order $\leq 1$ of functions in $L_{\bar{M}}(\Omega)\left(\right.$ resp. $\left.E_{\bar{M}}(\Omega)\right)$. It is a Banach space under the usual quotient norm.

If the open set $\Omega$ has the segment property, then the space $\mathcal{D}(\Omega)$ is dense in $W_{0}^{1} L_{M}(\Omega)$ for the modular convergence and thus for the topology $\sigma\left(\prod L_{M}, \prod L_{\bar{M}}\right)$ (cf. [13], [14]). Consequently, the action of a distribution in $W^{-1} L_{\bar{M}}(\Omega)$ on an element of $W_{0}^{1} L_{M}(\Omega)$ is well defined.

The following abstract lemma will be applied in the following.

Lemma 2.1. (see [6]) Let $F: \mathbf{R} \rightarrow \mathbf{R}$ be uniformly lipschitzian, with $F(0)=0$. Let $M$ be an $N$ - function and let $u \in W_{0}^{1} L_{M}(\Omega)$ (resp. $\left.W_{0}^{1} E_{M}(\Omega)\right)$.

Then $F(u) \in W_{0}^{1} L_{M}(\Omega)$ (resp. $W_{0}^{1} E_{M}(\Omega)$ ). Moreover, if the set of discontinuity points of $F^{\prime}$ is finite, then:

$$
\frac{\partial}{\partial x_{i}} F(u)= \begin{cases}F^{\prime}(u) \frac{\partial u}{\partial x_{i}} & \text { a.e. in }\{x \in \Omega: u(x) \notin D\} \\ 0 & \text { a.e. in }\{x \in \Omega: u(x) \in D\}\end{cases}
$$

\section{Main results}

Theorem 3.1. Under the hypotheses (1.1)', (1.2), (1.3)', (1.4), (1.5) $f \in L_{\text {loc }}^{1}\left(\mathbf{R}^{N}\right), \delta>p-1, p_{0}<p \leq N$ and $\alpha>\frac{1}{p}$, there exists at least one weak solution $u \in W_{\text {loc }}^{1, \bar{q}}\left(\mathbf{R}^{N}\right)$ for (1.7). 
A. BENKIRANE AND M. KBIRI ALAOUI EXISTENCE OF SOLUtions OF STRONGLY ...

In the following, we put $M(t)=t^{p} \log ^{\alpha p}(e+t)$, where $\left.\left.p \in\right] 1, N\right]$ and $\alpha>\frac{1}{p}$.

Recall that the N-function $M$ and $\bar{M}$ satisfy the $\Delta_{2}$ condition on $\mathbf{R}^{+}$ (see [17]).

\section{Proof of Theorem.}

For $n \geq 1$, we set $f_{n}=\inf (|f|, n) \operatorname{sign}(f), B_{n}=\left\{x \in \mathbf{R}^{N},|x|<n\right\}$. Consider the approximate problem :

$$
\left\{\begin{array}{l}
A\left(u_{n}\right)+g\left(., u_{n}\right)=f_{n} \\
u_{n} \in W_{0}^{1} L_{M}\left(B_{n}\right), g\left(., u_{n}\right) \in L^{1}\left(B_{n}\right), u_{n} g\left(., u_{n}\right) \in L^{1}\left(B_{n}\right) .
\end{array}\right.
$$

The problem (1.3) has a solution by theorem 6 of [15].

\section{Estimation}

Case $p=N$

Step 1. In the following, all constants $C, C_{i}$ and $C_{i}^{\prime}$ depends only on the data.

We follow the same argument as in lemma 2.1 of [11].

Let $K$ be the $\mathrm{N}$-function defined by $K(t)=\exp t^{N^{\prime}} \_1$. We recall that $W^{1, N}\left(B_{r}\right) \hookrightarrow L_{K}\left(B_{r}\right)$

Let $r>0, n>2 r$, we claim there exists a constants $C$ which does not depend on $n$ such that:

$$
\begin{gathered}
\left\|u_{n}\right\|_{\delta, B_{r}} \leq\left\|g\left(., u_{n}\right)\right\|_{1, B_{r}} \leq C . \\
\int_{B_{r}} \frac{M\left(\left|\nabla u_{n}\right|\right)}{\left(1+L_{n}\right) \log ^{\alpha N}\left(e+L_{n}\right)} \leq C\left\|u_{n}\right\|_{K, B_{r}}
\end{gathered}
$$

where $L_{n}=\frac{\left|u_{n}\right|}{\left\|u_{n}\right\|_{K, B_{r}}}$.

(3.3) if $\left\|u_{n}\right\|_{K, B_{r}}>1$, then

In the following, we omit the index $n$ for simplicity.

Let $\phi: \mathbf{R} \rightarrow \mathbf{R}$ the function defined by :

$$
\phi(s)= \begin{cases}\int_{0}^{s} \frac{d t}{(1+t) \log ^{\alpha N}(e+t)} & \text { if } s \geq 0 \\ -\phi(-s) & \text { if } s<0\end{cases}
$$


Let $\zeta \in \mathcal{D}\left(B_{2 r}\right), 0 \leq \zeta \leq 1, \zeta=1$ in $B_{r}$ and $|\nabla \zeta| \leq \frac{2}{r}$.

Let $\eta>0$ and $0<\epsilon<\frac{1}{2}$ (the choice of $\epsilon$ and $\eta$ will be fixed in the following).

We take $v=\phi(u) \zeta^{\eta}$ as test function in (3.1) (see lemma 2.1), we have

$$
\begin{aligned}
\int a(., u, \nabla u) \phi^{\prime}(u) \nabla u \zeta^{\eta}+ & \int g(., u) \phi(u) \zeta^{\eta} \leq \int f \phi(u) d x+\frac{2}{r} \int_{B_{2 r}} c(x) d x \\
& +\frac{2 \eta}{r} C \int|\nabla u|^{N-1} \log ^{\alpha N}(e+|\nabla u|) \phi(u) \zeta^{\eta-1} \\
& +\frac{2 \eta}{r} C \int|u|^{N-1} \log ^{\alpha N}(e+|u|) \phi(u) \zeta^{\eta-1}
\end{aligned}
$$

in the other hand, we have

$$
\begin{aligned}
& |\nabla u|^{N-1} \log ^{\alpha N}(e+|\nabla u|) \zeta^{\eta-1}= \\
& |\nabla u|^{N-1} \log ^{\alpha(N-1+\epsilon)}(e+|\nabla u|) \log ^{\alpha(1-\epsilon)}(e+|\nabla u|) \zeta^{\eta-1} \\
& \leq C(\alpha, \epsilon)|\nabla u|^{N-1+\epsilon} \log ^{\alpha(N-1+\epsilon)}(e+|\nabla u|) \zeta^{\eta-1} .
\end{aligned}
$$

Let $h>0, l=\frac{N}{N-1+\epsilon}$, by Young inequality with exponents $l, l^{\prime}=\frac{N}{N-\epsilon}$ we obtain

$$
\begin{aligned}
& |\nabla u|^{N-1} \log ^{\alpha N}(e+|\nabla u|) \zeta^{\eta-1} \\
& \leq C(\alpha, \epsilon, N) \zeta^{\eta} h \frac{M(|\nabla u|)}{(1+|u|) \log ^{\alpha N}(e+|u|)} \\
& +(1+|u|)^{\left(l^{\prime}-1\right)} \log ^{\alpha N\left(l^{\prime}-1\right)}(e+|u|) \zeta^{\eta-l^{\prime}} \frac{C(\alpha, \epsilon,)}{h^{l^{\prime}-1}} .
\end{aligned}
$$

Hence, by (1.3)'

$$
\begin{aligned}
& \nu \int M(|\nabla u|) \phi^{\prime}(u) \zeta^{\eta}+\int g(., u) \phi(u) \zeta^{\eta} \\
& \leq C_{1}+\frac{2 \eta}{r} C h \int M(|\nabla u|) \phi^{\prime}(u) \zeta^{\eta} \\
& +\frac{2 \eta C}{r h^{l^{\prime}-1}} \int(1+|u|)^{\left(l^{\prime}-1\right)} \log ^{\alpha N\left(l^{\prime}-1\right)}(e+|u|) \zeta^{\eta-l^{\prime}} \\
& +\frac{2 \eta}{r} C \int|u|^{N-1} \log ^{\alpha N}(e+|u|) \phi(u) \zeta^{\eta-1} d x,
\end{aligned}
$$

where $C=C(\alpha, \epsilon, N, r)$.

We shall fix $\epsilon>0$ such that $l^{\prime}-1<\delta$ and $\eta>l^{\prime} \frac{\delta}{\delta-\mu}$. 
We choose $\mu$, such that $N-1<\mu<\delta$, and $l^{\prime}-1<\mu<\delta$, then

$$
\begin{aligned}
\nu \int M(|\nabla u|) \phi^{\prime}(u) \zeta^{\eta} d x+ & \int g(., u) \phi(u) \zeta^{\eta} d x \\
\leq & C_{1}+C_{2} h \int M(|\nabla u|) \phi^{\prime}(u) \zeta^{\eta} d x \\
& +C_{2} \int(1+|u|)^{\mu} \zeta^{\eta-l^{\prime}} d x
\end{aligned}
$$

since the logarithm function is less than any power function small enough near infinity.

A convenient $h$ gives:

$$
\begin{aligned}
\nu^{\prime} \int M(|\nabla u|) \phi^{\prime}(u) \zeta^{\eta} d x+ & \int g(., u) \phi(u) \zeta^{\eta} d x \leq \\
& \leq C_{1}+\leq C_{2} \int(1+|u|)^{\mu} \zeta^{\eta-l^{\prime}} d x
\end{aligned}
$$

with $\nu^{\prime}>0$.

Let now $\lambda>0$. By using the Young inequality, with exponents $\frac{\delta}{\mu}$ and $\left(\frac{\delta}{\mu}\right)^{\prime}$, we have

$$
\zeta^{\eta-l^{\prime}}(1+|u|)^{\mu} \leq \frac{\lambda \mu \zeta^{\eta}}{\delta}(1+|u|)^{\delta}+\frac{\delta-\mu}{\delta \lambda^{\frac{\mu}{\delta-\mu}}} \zeta^{\eta-l^{\prime} \frac{\delta}{\delta-\mu}}
$$

$\eta$ is chosen such that, $\eta>l^{\prime} \frac{\delta}{\delta-\mu}$, hence

$$
\zeta^{\eta-l^{\prime}}(1+|u|)^{\mu} \leq \frac{2^{\delta} \lambda \mu \zeta^{\eta}}{\delta}|u|^{\delta}+\frac{2^{\delta} \lambda \mu \zeta^{\eta}}{\delta}+\frac{\delta-\mu}{\delta \lambda^{\frac{\mu}{\delta}-\mu}} \zeta^{\eta-l^{\prime}} \frac{\delta}{\delta-\mu}
$$

We choose $\lambda$ small such that $\frac{2^{\delta} \lambda \mu}{\delta}<1$.

We introduce (3.6) in (3.5) and we use the fact that $|s|^{\delta} \leq g(., s) \frac{\phi(s)}{\phi(1)}+1$, we obtain

$$
\nu^{\prime} \int_{B_{r}} M(|\nabla u|) \phi^{\prime}(u) \zeta^{\eta} d x+C \int_{B_{r}} g(., u) \phi(u) \zeta^{\eta} d x \leq C_{3}
$$


A. Benkirane AND M. KBIRI AlaOui Existence of SOlutions of Strongly ...

Then, we deduce (3.2), and

$$
\int_{B_{r}} M(|\nabla u|) \phi^{\prime}(u) \zeta^{\eta} d x \leq C
$$

In order to obtain (3.3), we proceed as follow:

In (3.1) we take $v=\phi_{0}(u)$ as test function, where $\phi_{0}(u)=$ $\|u\|_{K, B_{r}} \phi\left(\frac{u}{\|u\|_{K, B_{r}}}\right)$ and we suppose $\|u\|_{K, B_{r}}>1$.

We follow the same way as before, we have then in place of (3.5), the inequality

$$
\begin{aligned}
& \nu^{\prime} \int M(|\nabla u|) \phi^{\prime}\left(\frac{|u|}{\|u\|_{K, B_{r}}}\right) \zeta^{\eta} d x+\int g(., u) \phi_{0}(u) \zeta^{\eta} d x \leq C_{1}^{\prime}\|u\|_{K, B_{r}} \\
& +C_{2}^{\prime}\|u\|_{K, B_{r}} \int(1+|u|)^{\mu} \zeta^{\eta-l^{\prime}} d x
\end{aligned}
$$

since $\|u\|_{K, B_{r}}>1$

We now combine (1.5), (3.2) and (3.6) to get

$$
\nu^{\prime} \int M(|\nabla u|) \phi^{\prime}\left(\frac{|u|}{\|u\|_{K, B_{r}}}\right) \zeta^{\eta} d x \leq C_{1}^{\prime}\|u\|_{K, B_{r}}+C_{2}^{\prime}\|u\|_{K, B_{r}} .
$$

Then (3.3) follows.

Step 2. In this step, we give an estimate of the solution $u$ in $W^{1, N}\left(B_{r}\right)$. Let $H(t)=t \log ^{\alpha N}(e+t), B(t)=t \log (e+t)$.

Let $0<\epsilon<\frac{1}{2}, 1+\epsilon<N^{\prime}$ and $\log ^{\alpha^{N}}(e+t) \leq \frac{t^{\epsilon}}{\epsilon}$, for all $t \geq 1$.

W e suppose $L \geq 1$, then : 
A. BENKIRANE AND M. KBIRI AlAOUI EXISTENCE OF SOLUTIONS OF STRONGLY ...

$$
\begin{aligned}
\frac{\epsilon}{2}|\nabla u|^{N} & =\frac{\epsilon}{2} \frac{|\nabla u|^{N}}{(1+L) \log ^{\alpha^{N}}(e+L)}(1+L) \log ^{\alpha^{N}}(e+L) \\
& \leq H\left(\frac{|\nabla u|^{N}}{(1+L) \log ^{\alpha^{N}}(e+L)}\right)+\bar{H}\left(\epsilon L \log ^{\alpha^{N}}(e+L)\right) \\
& \leq C \frac{M(|\nabla u|)}{(1+L) \log ^{\alpha^{N}}(e+L)}+C+\bar{H}\left(L^{1+\epsilon}\right) \\
& \leq C \frac{M(|\nabla u|)}{(1+L) \log ^{\alpha^{N}}(e+L)}+C+K(L)
\end{aligned}
$$

since $\bar{H}(t)<\bar{B}(t)$ and $1+\epsilon<N^{\prime}$.

Thus

$$
|\nabla u|^{N} \leq C \frac{M(|\nabla u|)}{(1+L) \log ^{\alpha N}(e+L)}+C+C K(L)
$$

In the other case, where $L<1$, we obtain a similar inequality.

If $\|u\|_{K, B_{r}}>1$, the first step gives

$$
\int_{B_{r}}|\nabla u|^{N} d x \leq C\|u\|_{K, B_{r}}+C
$$

If $\|u\|_{K, B_{r}} \leq 1$, then by a similair argument as before, we have

$$
|\nabla u|^{N} \leq C \frac{M(|\nabla u|)}{(1+|u|) \log ^{\alpha N}(e+|u|)}+C+C K(|u|) .
$$

Also

$$
\int_{B_{r}}|\nabla u|^{N} d x \leq C\|u\|_{K, B_{r}}+C .
$$

In the both cases, we obtain

$$
\|\nabla u\|_{N, B_{r}}^{N} \leq C\|u\|_{K, B_{r}}+C .
$$

In the other hand, we have $\left\|u_{-} \bar{u}_{r}\right\|_{K, B_{r}} \leq C\|\nabla u\|_{N, B_{r}}$ (see [12]), where $\bar{u}_{r}=\frac{1}{\left|B_{r}\right|} \int_{B_{r}} u d x$

Then

$$
\|u\|_{K, B_{r}} \leq C\|\nabla u\|_{N, B_{r}}+\frac{1}{K^{-1}\left(\frac{1}{\left|B_{r}\right|}\right)}\left|\bar{u}_{r}\right| .
$$


A. BENKIRANE AND M. KBIRI AlAOUI EXISTENCE OF SOLUTIONS OF STRONGLY ...

Holder inequality gives

$$
\left|\bar{u}_{r}\right| \leq \frac{1}{\left|B_{r}\right|^{\frac{1}{N^{\prime}}}}\|u\|_{N^{\prime}, B_{r}}
$$

We suppose $\|u\|_{K, B_{r}}>1$ (the other case is obvious by (3.7)), then

$$
\|u\|_{N^{\prime}, B_{r}}^{N^{\prime}} \leq C\|u\|_{K, B_{r}}
$$

Indeed:

If $N>2$, then $N^{\prime}<2<\delta$ and $\|u\|_{N^{\prime}, B_{r}} \leq C\|u\|_{\delta, B_{r}} \leq C$, hence

$$
\|u\|_{N^{\prime}, B_{r}}^{N^{\prime}} \leq C\|u\|_{K, B_{r}}
$$

If $N=2, \int_{B_{r}}|u|^{2} \leq\|u\|_{\delta, B_{r}}\|u\|_{\delta^{\prime}, B_{r}} \leq C\|u\|_{K, B_{r}}$

By (3.2) and since $L_{K}\left(B_{r}\right) \hookrightarrow L^{\delta^{\prime}}\left(B_{r}\right)$ (see [1])

Then, $\|u\|_{N^{\prime}, B_{r}}^{N^{\prime}} \leq C\|u\|_{K, B_{r}}$, so

$$
\|u\|_{K, B_{r}} \leq C\|u\|_{K, B_{r}}^{\frac{1}{N}}+\frac{1}{K^{-1}\left(\frac{1}{\left|B_{r}\right|}\right)} \frac{1}{\left|B_{r}\right|^{\frac{1}{N^{\prime}}}}\|u\|_{K, B_{r}}^{\frac{1}{N^{\prime}}}
$$

We deduce then

$$
\|u\|_{K, B_{r}} \leq C, \text { also }\|\nabla u\|_{N, B_{r}} \leq C .
$$

hence

$$
\begin{aligned}
&\left\|g\left(., u_{n}\right)\right\|_{1, B_{r}} \leq C . \\
&\left\|u_{n}\right\|_{W^{1, N}\left(B_{r}\right)} \leq C .
\end{aligned}
$$

Case $p_{0}<p<N$.

As the same proof as in step 1, we have (3.2) and (3.3)' by taking $l=\frac{p}{(p-1)+\epsilon}$ with an appropriate $\epsilon$. 
We have with $\bar{q}^{*}=\frac{\bar{q}}{p-\bar{q}}$

$$
\begin{aligned}
& \int_{B_{r}}\left|\nabla u_{n}\right|^{\bar{q}} d x=\int_{B_{r}} \frac{\left|\nabla u_{n}\right|^{\bar{q}}}{\left(1+\left|u_{n}\right|\right)^{\frac{\bar{q}}{p}}}\left(1+\left|u_{n}\right|\right)^{\frac{\bar{q}}{p}} d x \\
\leq & \left(\int_{B_{r}} \frac{\left|\nabla u_{n}\right|^{p}}{\left(1+\left|u_{n}\right|\right)} d x\right)^{\frac{\bar{q}}{p}}\left(\int_{B_{r}}\left(1+\left|u_{n}\right|\right)^{\bar{q}^{*}} d x\right)^{1-\frac{\bar{q}}{p}} \\
\leq & \left(\int_{B_{r} \cap\left\{\left|u_{n}\right|<\left|\nabla u_{n}\right|\right\}} \frac{M\left(\left|\nabla u_{n}\right|\right)}{\left(1+\left|u_{n}\right|\right) \log ^{\alpha p}\left(e+\left|\nabla u_{n}\right|\right)}\right. \\
+ & \left.\int_{B_{r}}\left|\nabla u_{n}\right|^{p-1}\right)^{\frac{\bar{q}}{p}}\left(\int_{B_{r}}\left(1+\left|u_{n}\right|\right)^{\bar{q}^{*}}\right)^{1-\frac{\bar{q}}{p}} \\
\leq & C+C\left(\int_{B_{r}}\left|u_{n}\right|^{\bar{q}^{*}}\right)^{1-\frac{\bar{q}}{p}}
\end{aligned}
$$

by (3.3)' and since $\left\|\nabla u_{n}\right\|_{p-1, B_{r}} \leq C$ (lemma 2.2 of [11]).

Then, we continue the proof as in lemma 2.2 ([11] part i)) to have the boundedness of $\left(u_{n}\right)$ in $W^{1, \bar{q}}\left(B_{r}\right)$.

\section{Passage to the limit.}

By the estimation's step we have, $u_{n}$ is bounded in $W^{1, \bar{q}}\left(B_{r}\right)$, then there exists a subsequence noted $\left(u_{n}\right)$ such that:

$$
\begin{cases}u_{n} \rightarrow u & \text { weakly in } W^{1, \bar{q}}\left(B_{r}\right) \\ u_{n} \rightarrow u & \text { strongly in } L^{\bar{q}}\left(B_{r}\right)\end{cases}
$$

By the same technique as in lemma 1 of [10], we deduce that:

$$
\nabla u_{n} \rightarrow \nabla u \text { in measure and also a.e. }
$$

We have $g\left(., u_{n}\right) \rightarrow g(., u)$ a.e. and $\left(g\left(., u_{n}\right)\right)_{n \geq 0} \subset L_{l o c}^{1}\left(\mathbf{R}^{N}\right)$, then

$$
g\left(., u_{n}\right) \rightarrow g(., u) \text { strongly in } L_{l o c}^{1}\left(\mathbf{R}^{N}\right) .(\text { see }[11])
$$


We combine the fact that $a$ is a Carathéodory function, the boundedness of $\left(u_{n}\right)$ in $W_{l o c}^{1, \bar{q}}\left(\mathbf{R}^{N}\right)$ and the simple inequality: $\log (e+t) \leq C \frac{t^{\epsilon}}{\epsilon}$ for $t \geq 1, \epsilon \in] 0, \frac{1}{2}[$, we deduce the existence for some $\beta>1$ such that:

$$
a\left(., u_{n}, \nabla u_{n}\right) \rightarrow a(., u, \nabla u) \text { strongly in } L_{l o c}^{\beta}\left(\mathbf{R}^{N}\right) .
$$

By passage to the limit, we obtain that $u$ is a weak solution of (1.6) and $u \in W_{l o c}^{1, \bar{q}}\left(\mathbf{R}^{N}\right)$.

In the following, we suppose $a$ (resp. $g$ ) satisfies (1.1)', (1.2), (1.3)' (resp. (1.4), (1.5)).

Remark 3.1. In the last theorem, if $f$ is nonnegative, so is $u$ (see [7]).

Remark 3.2. The theorem 3.1 can be formulated as follow:

Under the hypotheses (1.1)', (1.2), (1.3)', (1.4), (1.5), $f \in L_{l o c}^{1}\left(\mathbf{R}^{N}\right), \delta>$ $p-1, \alpha>\frac{1}{p}$, there exists at least one weak solution $u \in W_{l o c}^{1, \bar{q}}\left(\mathbf{R}^{N}\right)$ for (1.6) satisfying, for every $r>0$

$$
\left\{\begin{array}{l}
\|u\|_{K, B_{r}} \leq C\|f\|_{1, B_{2 r}}+C(\text { in the case } p=N) \\
\|\nabla u\|_{\bar{q}, B_{r}} \leq C\|f\|_{1, B_{2 r}}+C
\end{array}\right.
$$

Remark 3.3. The condition $\alpha>\frac{1}{p}$ is necessary to obtain the regularity $W_{l o c}^{1, \bar{q}}\left(\mathbf{R}^{N}\right)$ for the solutions satisfying $(H)$.

Counterexample. We suppose $p=N=2,0<\alpha \leq \frac{1}{2}$ and for every $f \in L_{l o c}^{1}\left(\mathbf{R}^{N}\right)$, the problem (1.7) has a solution $u \in W_{l o c}^{1, \bar{q}}\left(\mathbf{R}^{N}\right)$ satisfying (H).

Let $\mu \in M_{b}\left(\mathbf{R}^{N}\right), A(u)=-\operatorname{div}\left(\nabla u \log ^{2 \alpha}(e+|\nabla u|)\right), g(., s)=|s|^{\delta-1} s, \delta>$ 1 and $K(t)=\exp t^{N^{\prime}}-1$.

Consider the problem :

$$
\left\{\begin{array}{l}
u \in W_{l o c}^{1, \bar{q}}\left(\mathbf{R}^{N}\right), a(., \nabla u) \in L_{l o c}^{1}\left(\mathbf{R}^{N}\right), g(., u) \in L_{l o c}^{1}\left(\mathbf{R}^{N}\right) \\
A(u)+g(., u)=\mu \text { in } \mathcal{D}^{\prime}\left(\mathbf{R}^{N}\right)
\end{array}\right.
$$

Let $\left(f_{n}\right) \subset W^{-1} L_{\bar{M}}\left(B_{2 r}\right) \cap L^{1}\left(B_{2 r}\right)$ such that $\left\|f_{n}\right\|_{1} \leq\|\mu\|_{M_{b}\left(B_{2 r}\right)}$ and $f_{n} \rightarrow \mu$ in $\mathcal{D}^{\prime}\left(B_{2 r}\right)$, for every $r>0$. There exists a solution $u_{n} \in$ 
$W_{l o c}^{1, \bar{q}}\left(\mathbf{R}^{N}\right)$ satisfying $(\mathrm{H})$ solution of the problem:

$$
\left\{\begin{array}{l}
u_{n} \in W_{l o c}^{1, \bar{q}}\left(\mathbf{R}^{N}\right), a\left(., \nabla u_{n}\right) \in L_{l o c}^{1}\left(\mathbf{R}^{N}\right), g\left(., u_{n}\right) \in L_{l o c}^{1}\left(\mathbf{R}^{N}\right) \\
A\left(u_{n}\right)+g\left(., u_{n}\right)=f_{n} \text { in } \mathcal{D}^{\prime}\left(\mathbf{R}^{N}\right)
\end{array}\right.
$$

$\left(u_{n}\right)$ is bounded in $L_{K}\left(B_{r}\right)$ and $\left(\left|\nabla u_{n}\right|\right)$ is bounded in $L^{\bar{q}}\left(B_{r}\right)$, so $\left(u_{n}\right)$ is in $W^{1, \bar{q}}\left(B_{r}\right)$.

A subsequence $\left(u_{n}\right)$ and $u \in W^{1, \bar{q}}\left(B_{r}\right)$ exist such that:

$$
\left\{\begin{array}{l}
u_{n} \rightarrow u \text { in } W^{1, \bar{q}}\left(B_{r}\right), \text { weakly and a.e. in } B_{r} \\
g\left(., u_{n}\right) \rightarrow g(., u) \text { a.e in } B_{r} .
\end{array}\right.
$$

$\left(u_{n}\right)$ is bounded in $L_{K}\left(B_{r}\right)$, so $\left(\left|u_{n}\right|^{\delta-1} u_{n}\right)$ is bounded in $L_{K^{\prime}}\left(B_{r}\right)$, with $K(t)=\exp t^{N^{\prime}}-1$ and $K^{\prime}(t)=\exp t^{\frac{\delta}{N^{\prime}}}-1$. Then the subsequence $\left(g\left(., u_{n}\right)\right)$ is equiintegrable on $B_{r}$, so

$$
g\left(., u_{n}\right) \rightarrow g(., u) \text { strongly in } L^{1}\left(B_{r}\right) .
$$

Then, we deduce $A(u)=\mu-g(., u)=\mu-\nu$ in $\mathcal{D}^{\prime}\left(B_{r}\right)$ and $u \in W_{l o c}^{1, \bar{q}}\left(\mathbf{R}^{N}\right)$. $\nu$ is a Radon measure associated to $g(., u)$ which does not charge the sets of zero $C_{1, N_{\alpha}}$, where $C_{1, N_{\alpha}}$ is the $N_{\alpha}$ - capacity defined in [3].

Let $r \in \mathbf{R}_{+}^{*}$ small enough $(0<r<1 / 2), f(x)=|x|^{-r}$, then:

$$
\begin{aligned}
& f \in L_{N_{\alpha}}\left(B_{r}\right), N_{\alpha}(t)=t^{2} \log ^{4 \alpha}(e+t) . \\
& G_{1} * f(0)=+\infty, G_{1} \text { is the Bessel potential. }
\end{aligned}
$$

Indeed, it is clear that $f \in L_{N_{\alpha}}\left(B_{r}\right)$, since $r$ is small.

$G_{1} * f(0)>\int_{B(0,1)} G_{1}(y) f\left({ }_{-} y\right) d y \geq C \int_{B(0, l)}|y|^{\vdash_{-}-1} d y=+\infty$, where $l$ is small enough, since $G_{1}$ is equivalent to $|y|^{-1}$ near zero.

By (3.9), (3.10) and theorem 3 of [3], we have

$$
C_{1, N_{\alpha}}\{0\}=0 .
$$

We have also $A(u) \in W^{-1} L_{\overline{N_{\alpha}}}\left(B_{r}\right)$ and $J\left(N_{\alpha}, 2\right) \geq 1$ (where $J\left(N_{\alpha}, 2\right)$ is the well known Donaldson-Trudinger's indice defined in [1] chap.8)

Indeed:

$\overline{N_{\alpha}}(t) \leq C t^{2} \log ^{-4 \alpha}(e+t)$ (see [17]), hence

$\overline{N_{\alpha}}(|a(., \nabla u)|) \leq C|\nabla u|^{2} \log ^{4 \alpha}(e+|\nabla u|) \log ^{-4 \alpha}(e+|\nabla u|$ 
$\left.\log ^{2 \alpha}(e+|\nabla u|)\right) \leq C|\nabla u|^{2}$.

On the other hand, since $N_{\alpha}^{-1}(t) \geq C t^{\frac{1}{2}} \log ^{-2 \alpha}(e+t)$, then $\int_{\text {. }}^{\infty} \frac{N_{\alpha}^{-1}(t)}{t^{1+\frac{1}{2}}} d t=$ $+\infty$ (because $\alpha \leq \frac{1}{2}$ ).

Then, we conclude : $C_{1, N_{\alpha}}\{0\}=0, A(u) \in W^{-1} L_{\overline{N_{\alpha}}}\left(B_{r}\right) \cap M_{b}\left(B_{r}\right)$ and $J\left(N_{\alpha}, 2\right) \geq 1$.

By lemma 2 of [4] (see also [2]), $A(u)$ defines a bounded measure which is absolutely continuous with respect to $C_{1, N_{\alpha}}$, then if we take $\mu=\delta_{0}$ we deduce the result.

Remark 3.4. In the case $p=N, \alpha>\frac{1}{N}, g(., s)=|s|^{\delta-1} s$ and $\delta>$ $N-1$, (3.8) has a weak solution $u \in W_{l o c}^{1, \bar{q}}\left(\mathbf{R}^{N}\right)$.

Indeed:

Let $r, f_{n}, u_{n}$ as in the counterexample. The subsequence $\left(g\left(., u_{n}\right)\right)$ is equiintegrable on $B_{r}$, so

$g\left(., u_{n}\right) \rightarrow g(., u)$ strongly in $L^{1}\left(B_{r}\right)$.

We then conclude the result.

Theorem 3.2. Let $1<p \leq p_{0}, \delta(p-1)>1$, and $\alpha>\frac{1}{p}$. Then, for every $f \in L_{\text {loc }}^{1}\left(\mathbf{R}^{N}\right)$, there exists at least a weak solution $u$ of (1.6), which belongs to $W_{l o c}^{1, q_{1}}\left(\mathbf{R}^{N}\right)$.

Proof. We take the same constructions of the solutions $u_{n}$ as in theorem 3.1.

By the same technique as in step 1 (theorem 3.1), we have (3.2), (3.3)', which we combine with lemma 2.3 of [11], Young inequality and using the decomposition:

$B_{r}=\left(\left\{\left|u_{n}\right|<\left|\nabla u_{n}\right|\right\} \cap B_{r}\right) \cup\left(\left\{\left|u_{n}\right|\left|\nabla u_{n}\right|\right\} \cap B_{r}\right)$, we obtain

$$
\begin{aligned}
\int_{B_{r}}\left|\nabla u_{n}\right|^{q_{1}} d x & \leq \int_{B_{r}} \frac{\left|\nabla u_{n}\right|^{p}}{1+\left|u_{n}\right|} d x+C \int_{B_{r}}\left(1+\left|u_{n}\right|\right)^{\delta} d x \\
& \leq \int_{B_{r}} \frac{M\left(\left|\nabla u_{n}\right|\right)}{\left(1+\left|u_{n}\right|\right) \log ^{\alpha p}\left(e+\left|u_{n}\right|\right)} d x \\
& +\int_{B_{r}}\left|\nabla u_{n}\right|^{p-1}+C \int_{B_{r}}\left|u_{n}\right|^{\delta} d x \leq C
\end{aligned}
$$


Since $\delta>p-1$, we have $q_{1}<\delta$, then $\left\|u_{n}\right\|_{q_{1}, B_{r}} \leq C$.

Then, we deduce:

$$
\left\{\begin{array}{l}
u_{n} \rightarrow u \text { weakly in } W^{1, q_{1}}\left(B_{r}\right) \\
u_{n} \rightarrow u \text { strongly in } L^{q_{1}}\left(B_{r}\right) \\
\left.g\left(., u_{n}\right) \rightarrow g(., u) \text { strongly in } L_{l o c}^{1}\left(\mathbf{R}^{N}\right) \text { (as in theorem } 3,[11]\right)
\end{array}\right.
$$

Following the same way as the third step (theorem 3.1), we conclude the result.

Theorem 3.3. Let $f \in L_{l o c}^{1}\left(\mathbf{R}^{N}\right)$, and assume that: $p_{0}<p<N, \delta>\bar{q}^{*}$ and $\alpha>\frac{1}{p}$, then the solution constructed in theorem 3.1 satisfies $|\nabla u| \in$ $L_{l o c}^{q_{1}}\left(\mathbf{R}^{N}\right)$.

Proof. As in theorem 3.1, we obtain

$$
\begin{aligned}
\int_{B_{r}}\left|\nabla u_{n}\right|^{q_{1}} d x & \leq \int_{B_{r}} \frac{M\left(\left|\nabla u_{n}\right|\right)}{\left(1+\left|u_{n}\right|\right) \log ^{\alpha p}\left(e+\left|\nabla u_{n}\right|\right)} d x \\
& +\int_{B_{r}}\left|\nabla u_{n}\right|^{p-1}+C \int_{B_{r}}\left|u_{n}\right|^{\delta} d x+C
\end{aligned}
$$

Combining (3.2), (3.3)', lemma 2.2 of [11] and using the same decomposition of $B_{n}$ as in theorem 3.2, we conclude the result.

Remark 3.5. It is to be noticed that the results in the last theorems can be proved in the case where we replace $\mathbf{R}^{N}$ by a bounded open subset $\Omega$. The proof is the same as in [11]. In this case, our existence result in theorem 3.1 is in some sense the "dual" of the following one (due to L. Boccardo and T. Gallouet in [10]): If the right hand side belongs to the Orlicz space $\operatorname{L} \log L(\Omega)$, then the solutions belong to $W_{0}^{1, \bar{q}}(\Omega)$.

\section{References}

[1] R. Adams, Sobolev spaces, Ac. Press, New York, 1975.

[2] N. Aissaoui, Bessel potentials in Orlicz spaces. Revista Matematica Complutense vol. $10 n^{0} 1$ (1997).

[3] N. Aissaoui et A. Benkirane, Capacités dans les espaces d'Orlicz, Ann.Sci.Math.Québec 18 (1994), 1-23. 
A. BENKIRANE AND M. KBIRI AlAOUI EXISTENCE OF SOLUTIONS OF STRONGLY ...

[4] A. Benkirane, A Theorem of H. Brezis and F. E. Browder type in Orlicz spaces and application, Pitman Research Notes in Mathematics series 343 (1994), pp. 10-36.

[5] A. Benkirane and J. P. Gossez, An approximation theorem for higher order Orlicz-Sobolev spaces, Studia Math., 92 (1989), 231-255.

[6] A. Benkirane and A. Elmahi, Almost everywhere convergence of the gradients of solutions to elliptic equations in Orlicz spaces and application, Nonlinear Anal. T. M. A. vol 28, $N^{0}$ 11, pp. 1769-1784, 1997.

[7] A. Benkirane and A. Elmahi, An existence theorem for a strongly nonlinear elliptic problem in Orlicz spaces, Nonlinear Anal.(1999), pp. 11-24.

[8] A. Benkirane and M. Kbiri Alaoui, On some nonlinear elliptic equations with right hand side measures-Preprint.

[9] L. Boccardo and T. Gallouet, Nonlinear elliptic and parabolic equations involving measure data, J. Funct. Anal. 87 (1989), 149-169.

[10] L. Boccardo and T. Gallouet, Nonlinear elliptic equations with right hand side measures, Comm. in Partial Diff. Equa., 17 (3 \& 4), (1992), 641-655.

[11] L. Boccardo, T. Gallouet and J. L. Vázquez, Nonlinear elliptic equations in $\mathbf{R}^{N}$ without growth restrictions on the second member, J. Diff. Equa. 105, 334-363 (1993).

[12] A. Cianchi, Continuity properties of functions from Orlicz-Sobolev spaces and embeddings theorems Ann. Scuola Norm. Sup. Pisa cl. Sci. (4 ) 23 (1996) $N^{0} 3,575-608$.

[13] J.-P. Gossez, some approximation properties in Orlicz-Sobolev spaces, Studia Math., 74 (1982), pp. 17-24.

[14] J.-P. Gossez, Nonlinear elliptic boundary value problems for equations with rapidely or slowly)increasing coefficients, Trans. Amer. Math. Soc. 190 (1974), 163-205.

[15] J.-P. Gossez, A strongly nonlinear elliptic problem in Orlicz-Sobolev spaces, Proc. A. M. S. Symp. Pure Math., 45 (1986), pp. 455.

[16] J.-P. Gossez and V. Mustonen, Variationnal inequality in Orlicz-Sobolev spaces, Nonlinear Anal. Theory Appli. 11 (1987), 379-392.

[17] M. Krasnoselśkii and Ya. Rutickii, convex functions and Orlicz spaces, Nodhoff Groningen, 1969. 
A. BENKIRANE AND M. KBIRI ALAOUi EXISTENCE OF SOLUTIONS OF STRONGLY ...

Département de Mathematiques

Faculté des Sciences Dhar El Mehraz

B.P. 1796 Atlas, Fès-Maroc

E-mail: benkirane@fesnet.net.ma

Recibido: 15 de Septiembre de 1998

Revisado: 16 de Febrero de 2000 